\title{
Clinicopathologic Features Associated With Having Four or More Metastatic Axillary Nodes in Breast Cancer Patients With a Positive Sentinel Lymph Node
}

\author{
Aeisha K. Rivers, MD, ${ }^{1,2}$ Kent A. Griffith, MS, MPH,${ }^{2}$ Kelly K. Hunt, MD, ${ }^{3}$ \\ Amy C. Degnim, MD, ${ }^{4}$ Michael S. Sabel, MD ${ }^{5}$ Kathleen M. Diehl, MD ${ }^{5}$ \\ Vincent M. Cimmino, MD, ${ }^{5}$ Alfred E. Chang, MD,${ }^{5}$ Peter C. Lucas, MD,${ }^{6}$ and \\ Lisa A. Newman, MD, MPH, FACS ${ }^{5}$
}

\footnotetext{
${ }^{1}$ Department of Surgery, St. Joseph’s Hospital and Medical Center, University of Michigan, 1500 East Medical Center Drive, Ann Arbor, Michigan 48109

${ }^{2}$ Department of Biostatistics, University of Michigan Comprehensive Cancer Center, University of Michigan, 1500 East Medical Center Drive, Ann Arbor, Michigan 48109

${ }^{3}$ Department of Surgery, University of Texas M. D. Anderson Cancer Center, 1515 Holcombe Boulevard, Houston, Texas 77030 ${ }^{4}$ Department of Surgery, Mayo Clinic, 200 First Street SW, Rochester, Minnesota 55905

${ }^{5}$ Department of Surgery, University of Michigan Comprehensive Cancer Center 1500 E. Medical Center Drive, 3308 Cancer Center, Ann Arbor, Michigan 48109-0932

${ }^{6}$ Department of Pathology, University of Michigan Comprehensive Cancer Center, University of Michigan, 1500 East Medical Center Drive, Ann Arbor, Michigan 48109
}

Received March 15, 2005; accepted August 1, 2005; published online January 1, 2006.

Address correspondence and reprint requests to: Lisa A. Newman, MD, MPH, FACS; E-mail: lanewman@umich.edu.

Published by Springer Science+Business Media, Inc. ㅇ 2006 The Society of Surgical Oncology, Inc.
Background: The survival benefit of a completion axillary lymph node dissection (ALND) in patients after removal of a metastatic sentinel lymph node (SLN) is uncertain and is under study in ongoing clinical trials. The completion ALND remains necessary, however, for the identification of cases with at least four metastatic lymph nodes, in which extended-field locoregional and/or postmastectomy radiation will be recommended. Our goal was evaluate clinicopathologic features that might serve as surrogates for determining which patients with a positive SLN are likely or unlikely to belong to this high-risk subset.

Methods: Records were reviewed for 285 patients from 2 comprehensive cancer centers who underwent completion ALND after resection of a metastatic SLN from 1995 to 2002. Clinicopathologic features were analyzed by univariate and multivariate logistic regression. Fortyone cases (14\%) were found to have at least four positive nodes after ALND.

Results: Fisher's exact test revealed the following features to be significantly $(P<.05)$ associated with having four or more nodal metastases: tumor size $>2 \mathrm{~cm}$, lymphovascular invasion, an increasing ratio of positive SLNs to the total number of resected SLNs, extranodal extension, and the size of the SLN metastasis. Patients whose largest SLN metastasis was $<2 \mathrm{~mm}$ had only a $1.4 \%$ risk of having four or more metastatic nodes $(P<.0001)$.

Conclusions: We conclude that patients with SLN micrometastases face an extremely low likelihood of having extensive nodal disease on completion ALND. Patients with larger primary tumors, lymphovascular invasion, and extranodal extension are more likely to have ALND findings that will affect their cancer management.

Key Words: Breast cancer-Sentinel lymph node metastases-Axillary lymph node dissection-Prediction of risk. 
definitive proof of axillary node-negative versus node-positive disease and provides excellent, durable control of regional disease. The extent to which an ALND contributes to breast cancer survival, however, is uncertain. The prognostic and staging benefits of the ALND must be weighed against its morbidity, including lymphedema, sensory disturbances, and shoulder dysfunction.

Intraoperative lymphatic mapping has therefore rapidly emerged as a primary approach to staging the axilla, and many institutions have come to accept a negative sentinel lymph node (SLN) as an accurate means of identifying the node-negative patient, for whom the ALND can be safely avoided. A completion ALND remains the standard of care for a patient with a metastatic SLN; however, this practice has been questioned because of studies demonstrating that axillary metastases are limited to the SLNs in $30 \%$ to $50 \%$ of cases. Furthermore, historic clinical trial data suggest that prophylactic resection of occult axillary metastases is comparable to axillary observation and delayed therapeutic ALND for cases of regional failure. ${ }^{1}$

The American College of Surgeons Oncology Group attempted to define the value of the ALND in node-positive breast cancer by randomizing SLN-positive patients to axillary observation versus the standard completion axillary operation. ${ }^{2}$ Unfortunately, this trial was recently closed because of poor accrual rates. ${ }^{3}$ The inability to complete this important trial is likely to strengthen interest in statistical models that can identify patients likely to harbor additional metastatic nodes after resection of at least one metastatic SLN. ${ }^{4,5}$ The goal of these prediction tools is to refine the selection of SLNpositive patients who require the completion ALND.

Given the uncertainties regarding the survival benefits associated with the completion ALND, a valid argument can be made that the critical issue is to identify the subset of high-risk SLN-positive patients, as defined by having a total of four or more metastatic lymph nodes, rather than identifying the subset of patients with any metastatic non-SLNs. It is important to accurately identify this subset of patients because they will be recommended to receive more extensive locoregional therapy in the form of postmastectomy radiation or extended-field radiation after lumpectomy. ${ }^{6,7}$ Available data suggest that this category represents $10 \%$ to $15 \%$ of all clinically earlystage breast cancer patients with at least one metastatic SLN. ${ }^{8-11}$ The goal of this study was to identify clinicopathologic features that distinguish SLN-po- sitive patients who are likely to have at least four metastatic axillary lymph nodes.

\section{MATERIALS AND METHODS}

The study population included SLN-positive breast cancer patients with $\mathrm{T} 1$ and $\mathrm{T} 2$ tumors (based on clinical assessment) who underwent completion ALND at 1 of 2 comprehensive cancer centers: the University of Michigan (103 cases) and the University of Texas M. D. Anderson Cancer Center (182 cases) between the years 1995 and 2002. Patients with four or more metastatic SLNs, patients who received neoadjuvant chemotherapy, and patients with insufficient information available on multiple clinicopathologic features were excluded from analysis. The conduct of these analyses was approved by the University of Michigan Institutional Review Board.

Clinicopathologic features for the study population were analyzed after stratification by extent of total axillary metastatic burden (a total of one to three positive nodes vs. four or more positive nodes).The significance of bivariate associations between patient, tumor, and SLN characteristics was explored by using the Fisher's exact test. Characteristics with $\mathrm{P}$ values $\leq 5 \%$ were considered for multivariate analysis. Unconditional logistic regression was used to model the occurrence of a positive case (having four or more metastatic nodes). For the computation of predicted probabilities, we chose to fix the total number of SLNs removed at the value of three, which is the median number of SLNs identified and resected. We applied this strategy to emphasize the contribution of the absolute number of metastatic SLNs, because the proportion represented by comparing the number of metastatic SLNs with the total number of SLNs resected will vary substantially with differences in the sentinel nodal drainage pattern and surgical specimen. Odds ratios and their $95 \%$ confidence intervals were reported for all characteristics found to be significant in the multivariate model.

\section{RESULTS}

Table 1 demonstrates the clinicopathologic features of the study populations. Forty-one (14\%) of the 285 evaluated cases were found to have at least 4 positive lymph nodes after completion ALND. The study population profiles from the two participating institutions were comparable, with an average age of 
TABLE 1. Patient, tumor, and node characteristics

\begin{tabular}{|c|c|c|c|}
\hline Characteristic & UMCC & MDACC & Total \\
\hline No. of cases & $103(36.1 \%)$ & $182(63.9 \%)$ & 285 \\
\hline \multicolumn{4}{|l|}{ Age } \\
\hline Median, y (range) & $52(31-78)$ & $52(29-78)$ & $52(29-78)$ \\
\hline$\leq 50, \mathrm{n}(\%)$ & $46(44.7)$ & 83 (45.6) & $129(45.3)$ \\
\hline$>50, \mathrm{n}(\%)$ & $57(55.3)$ & $99(54.4)$ & $156(54.7)$ \\
\hline \multicolumn{4}{|l|}{ Tumor histological findings, n (\%) } \\
\hline Ductal & $80(77.7)$ & $132(72.5)$ & $212(74.4)$ \\
\hline Lobular & $14(13.6)$ & $14(7.7)$ & $28(9.8)$ \\
\hline Ductal and lobular & $2(1.9)$ & $17(9.3)$ & $19(6.8)$ \\
\hline $\mathrm{DCIS}^{a}$ & $4(3.9)$ & $2(1.1)$ & $6(2.1)$ \\
\hline Other & $3(2.9)$ & $17(9.3)$ & $20(7.0)$ \\
\hline \multicolumn{4}{|l|}{ Tumor size $(\mathrm{cm}), \mathrm{n}(\%)$} \\
\hline$\leq 2$ & $69(67.0)$ & $116(63.7)$ & $185(64.9)$ \\
\hline$>2$ & $32(31.1)$ & $66(36.3)$ & $98(34.4)$ \\
\hline Unknown & $2(1.9)$ & 0 & $2(.7)$ \\
\hline \multicolumn{4}{|l|}{ Lymphovascular invasion, n (\%) } \\
\hline Present & $28(27.2)$ & $49(26.9)$ & $77(27.0)$ \\
\hline Absent & $73(70.9)$ & $133(73.1)$ & $206(72.3)$ \\
\hline Unknown & $2(1.9)$ & 0 & $2(.7)$ \\
\hline \multicolumn{4}{|l|}{ Extranodal extension, n (\%) } \\
\hline Present & $17(16.5)$ & $53(29.1)$ & $70(24.6)$ \\
\hline Absent & $78(75.7)$ & $129(70.9)$ & $207(72.6)$ \\
\hline Unknown & $8(7.8)$ & 0 & $8(2.8)$ \\
\hline \multicolumn{4}{|l|}{ Tumor grade, n (\%) } \\
\hline I & $16(15.5)$ & $15(8.2)$ & $31(10.9)$ \\
\hline II & $60(58.3)$ & $109(59.9)$ & $169(59.3)$ \\
\hline III & $20(19.4)$ & $55(30.2)$ & $75(26.3)$ \\
\hline Other/unknown & $7(6.8)$ & $3(1.7)$ & $10(3.5)$ \\
\hline \multicolumn{4}{|l|}{ Estrogen receptor, n (\%) } \\
\hline Positive & $78(75.7)$ & $150(82.4)$ & $228(80.0)$ \\
\hline Negative & $23(22.3)$ & $31(17.0)$ & $54(19.0)$ \\
\hline Unknown & $2(1.9)$ & $1(.6)$ & $3(1.1)$ \\
\hline Total lymph nodes removed, median (range) & $18(6-44)$ & $19(4-44)$ & $19(4-44)$ \\
\hline Non-SLNs removed, median (range) & $15(5-40)$ & $17(1-42)$ & $16(1-42)$ \\
\hline \multicolumn{4}{|l|}{ SLNs removed } \\
\hline Median (range) & $3(1-15)$ & $2(1-9)$ & $3(1-15)$ \\
\hline $1, \mathrm{n}(\%)$ & $22(21.4)$ & $38(20.9)$ & $60(21.1)$ \\
\hline $2, \mathrm{n}(\%)$ & $24(23.3)$ & $55(30.2)$ & $79(27.7)$ \\
\hline $3, \mathrm{n}(\%)$ & $28(27.2)$ & $42(23.1)$ & $70(24.6)$ \\
\hline$\geq 4, \mathrm{n}(\%)$ & $29(28.2)$ & $47(25.8)$ & $76(26.7)$ \\
\hline \multicolumn{4}{|l|}{ Positive SLNs, n (\%) } \\
\hline 1 & $73(70.9)$ & $125(68.7)$ & $198(69.5)$ \\
\hline 2 & $25(24.3)$ & $45(24.7)$ & $70(24.6)$ \\
\hline 3 & $5(4.9)$ & $12(6.6)$ & $17(6.0)$ \\
\hline \multicolumn{4}{|l|}{ Size of largest metastatic SLN focus, n (\%) } \\
\hline$<2 \mathrm{~mm}$ or IHC only & $38(36.9)$ & $36(19.8)$ & $74(26.0)$ \\
\hline$\geq 2 \mathrm{~mm}$ & $57(55.3)$ & $137(75.3)$ & $194(68.1)$ \\
\hline Unknown & $8(7.8)$ & $9(5.0)$ & $17(6.0)$ \\
\hline \multicolumn{4}{|l|}{ Total positive axillary lymph nodes } \\
\hline$<4$ & $89(86.4)$ & $155(85.2)$ & $244(85.6)$ \\
\hline$\geq 4$ & $14(13.6)$ & $27(14.8)$ & $41(14.4)$ \\
\hline
\end{tabular}

UMCC, University of Michigan Comprehensive Cancer Center; MDACC, M. D. Anderson Comprehensive Cancer Center; DCIS, ductal carcinoma-in-situ; SLN, sentinel lymph node; IHC, immunohistochemically detected.

${ }^{a}$ Predominantly DCIS histopathological findings but with suspected microinvasion.

52 years. The median number of SLNs removed was 3 , and the median number of non-SLNs resected with the completion ALND was 16. Approximately two thirds of the cases from both participating institutions were $\mathrm{T} 1$ cancers, with a median size of $18 \mathrm{~mm}$, and only three patients had T3 tumors (measuring 52, 60, and $67 \mathrm{~mm}$ on final pathologic analysis).
As shown by the bivariate comparisons presented in Table 2, factors found to be significantly associated with having four or more nodal metastases included a primary tumor size $>2 \mathrm{~cm}$, lymphovascular invasion, extranodal extension, the size of the SLN metastasis, and an increasing ratio of positive SLNs to the total number of resected SLNs. Patient age, tumor histological characteristics, tumor grade, and 
TABLE 2. Bivariate associations of patient/tumor/sentinel node characteristics with the extent of nodal metastasis

\begin{tabular}{|c|c|c|c|}
\hline Characteristic & $\begin{array}{l}\geq 4 \text { metastatic nodes } \\
(\mathrm{n}=41), \mathrm{n}(\%)\end{array}$ & $\begin{array}{l}1-3 \text { metastatic nodes } \\
(\mathrm{n}=244), \mathrm{n}(\%)\end{array}$ & $\begin{array}{l}\text { Fisher's exact } \\
P \text { value }\end{array}$ \\
\hline \multicolumn{4}{|l|}{ Age (y) } \\
\hline$\leq 50$ & $22(17.1)$ & $107(82.9)$ & \multirow[t]{2}{*}{.3090} \\
\hline$>50$ & $19(12.2)$ & $137(87.8)$ & \\
\hline \multicolumn{4}{|c|}{ Tumor histological findings } \\
\hline Ductal & $28(13.2)$ & $184(86.8)$ & \multirow[t]{5}{*}{.5816} \\
\hline Lobular & $5(17.9)$ & $23(82.1)$ & \\
\hline Ductal and lobular & $4(21.1)$ & $15(78.9)$ & \\
\hline DCIS & 0 & $6(100)$ & \\
\hline Other & $4(20.0)$ & $16(80.0)$ & \\
\hline \multicolumn{4}{|l|}{ Tumor size $(\mathrm{cm})$} \\
\hline$\leq 2$ & $15(8.1)$ & $170(91.9)$ & \multirow[t]{3}{*}{$<.0001$} \\
\hline$>2$ & $25(25.5)$ & $73(74.5)$ & \\
\hline Unknown & $1(50.0)$ & $1(50.0)$ & \\
\hline \multicolumn{4}{|c|}{ Lymphovascular invasion } \\
\hline Present & $21(27.3)$ & $56(72.7)$ & \multirow[t]{3}{*}{$<.0001$} \\
\hline Absent & $20(9.7)$ & $186(90.3)$ & \\
\hline Unknown & 0 & $2(100)$ & \\
\hline \multicolumn{4}{|l|}{ Extranodal extension } \\
\hline Present & $22(31.4)$ & $48(68.6)$ & \multirow[t]{3}{*}{$<.0001$} \\
\hline Absent & $19(9.2)$ & $188(90.8)$ & \\
\hline Unknown & 0 & $8(100)$ & \\
\hline \multicolumn{4}{|l|}{ Tumor grade } \\
\hline I & $1(3.2)$ & $30(96.8)$ & \multirow[t]{4}{*}{.2340} \\
\hline II & $26(15.4)$ & $143(84.6)$ & \\
\hline III & $13(17.3)$ & $62(82.7)$ & \\
\hline Other/unknown & $1(10.0)$ & $9(90.0)$ & \\
\hline \multicolumn{4}{|l|}{ Estrogen receptor } \\
\hline Positive & $31(13.6)$ & $197(86.4)$ & \multirow{3}{*}{.3457} \\
\hline Negative & $9(16.7)$ & $45(83.3)$ & \\
\hline Unknown & $1(33.3)$ & $2(66.7)$ & \\
\hline \multicolumn{4}{|l|}{ SLNs removed } \\
\hline 1 & $6(10.0)$ & $54(90.0)$ & \multirow[t]{4}{*}{.0079} \\
\hline 2 & $21(26.6)$ & $58(73.4)$ & \\
\hline 3 & $7(10.0)$ & $63(90.0)$ & \\
\hline$\geq 4$ & $7(9.2)$ & $69(90.8)$ & \\
\hline \multicolumn{4}{|l|}{ Positive SLNs } \\
\hline 1 & $10(5.1)$ & $188(94.9)$ & \multirow[t]{3}{*}{$<.0001$} \\
\hline 2 & $23(32.9)$ & $47(67.1)$ & \\
\hline 3 & $8(47.1)$ & $9(52.9)$ & \\
\hline \multicolumn{4}{|c|}{ Percentage of positive SLNs (\%) } \\
\hline $1-25$ & 0 & $46(100)$ & \multirow{4}{*}{$<.0001$} \\
\hline $26-50$ & $8(6.8)$ & $109(93.2)$ & \\
\hline $51-75$ & 7 (26.9) & $19(73.1)$ & \\
\hline $76-100$ & $26(27.1)$ & $70(72.9)$ & \\
\hline \multicolumn{4}{|c|}{ Size of largest metastatic SLN focus (mm) } \\
\hline$<2$ or IHC only & $1(1.4)$ & $73(98.6)$ & \multirow[t]{3}{*}{$<.0001$} \\
\hline$\geq 2$ & $36(18.6)$ & $158(81.4)$ & \\
\hline Unknown & $4(23.5)$ & $13(76.5)$ & \\
\hline
\end{tabular}

DCIS, ductal carcinoma-in-situ; SLN, sentinel lymph node; IHC, immunohistochemically detected.

hormone receptor status were not found to be significant predictors of an extensive metastatic nodal burden. The single feature associated with the lowest risk of having four or more metastatic axillary nodes was the size of the largest metastatic focus: of 74 patients with SLN micrometastases (tumor focus up to $2 \mathrm{~mm}$ in diameter), only $1(1.4 \%)$ was found to have high-risk nodal disease. Seventeen patients (which included four positive cases) in this dataset did not have any information for the size of their nodal disease. Not surprisingly, nearly half of patients with three metastatic SLNs had at least one additional positive non-SLN, thereby placing them into the high-risk subset. Only 5\% of patients with a single metastatic SLN were ultimately found to have four or more positive nodes.

Multivariate analysis was conducted by using the significant characteristics described previously. Tumor size, lymphovascular invasion, extranodal extension, the number of positive lymph nodes, and the ratio of positive SLNs to the total number of SLNs removed were all found to be independently 
TABLE 3. Multivariate associations of significant patient/tumor/SLN characteristics with high-risk axillary disease

\begin{tabular}{|c|c|c|c|c|}
\hline Characteristic & Estimate & OR & $95 \% \mathrm{CI}$ & $P$ value \\
\hline Intercept & -6.08 & & & \\
\hline \multicolumn{5}{|l|}{ Tumor size $(\mathrm{cm})$} \\
\hline$\leq 2$ & 0 & 1 & & \\
\hline$>2$ & 1.17 & 3.24 & $1.38-7.61$ & .0071 \\
\hline \multicolumn{5}{|l|}{ Lymphovascular invasion } \\
\hline Absent & 0 & 1 & & \\
\hline Present & 1.05 & 2.85 & $1.23-6.62$ & .0148 \\
\hline \multicolumn{5}{|l|}{ Extranodal extension } \\
\hline Absent & 0 & 1 & & \\
\hline Present & 1.08 & 2.94 & $1.24-6.96$ & .0141 \\
\hline \multicolumn{5}{|l|}{ Positive SLNs } \\
\hline 1 & 0 & 1 & & \\
\hline 2 & 1.47 & 4.35 & $1.76-10.75$ & \\
\hline 3 & 2.03 & 7.60 & $2.02-28.61$ & .0010 \\
\hline \multicolumn{5}{|c|}{ Percentage of positive SLNs } \\
\hline Continuous covariate & .03 & 1.03 & $1.01-1.05$ & .0001 \\
\hline
\end{tabular}

SLN, sentinel lymph node; OR, odds ratio; CI, confidence interval.

and significantly related to a final pathologic result of at least four metastatic nodes (Table 3). The size of the largest metastatic focus within the positive SLNs lost statistical significance after adjustment for the other characteristics in the multivariate analysis. Tumors $>2 \mathrm{~cm}$, lymphovascular invasion, and extranodal extension were all associated with an approximately 3 -fold increase in the odds of being a positive case. Having two or three positive SLNs, when compared with having only a single positive node, was associated with more than a 4- and 7-fold increase in the odds of being a positive case in this sample, respectively. Similarly, the likelihood of having extensive nodal disease was directly correlated with the ratio of positive SLNs to the total number of resected SLNs.

The predicted probabilities of having extensive nodal disease were calculated on the basis of the multivariate model in Table 3, and these data are reported in Table 4. Probabilities were calculated for a patient with one, two, or three positive SLNs, with the total number of SLNs fixed at 3, with and without the other risk factors. For example, Table 4 shows that a patient with two metastatic SLNs, a T2 or T3 tumor, and extranodal extension, but no lymphovascular invasion, has a $41.9 \%$ predicted probability of having high-risk nodal disease. The minimum predicted probability was an estimate of $.2 \%$ for cases of a single metastatic SLN with a T1 breast tumor that was negative for lymphovascular invasion and extranodal extension. The highest estimate, of $90 \%$, was calculated for cases of three metastatic SLNs associated with a tumor $>2 \mathrm{~cm}$ and characterized by both adverse histological patterns. For all cases of a single metastatic SLN, there was a $\leq 5 \%$ risk of having high-risk disease unless the primary tumor was $>2$ $\mathrm{cm}$ and both extranodal extension and lymphovascular invasion were present. With the latter combination, the risk of having high-risk disease increased to $15 \%$. All combinations of features that included three positive SLNs were associated with an at least $25 \%$ risk of having four or more metastatic nodes.

\section{DISCUSSION}

During the 1970s, the National Surgical Adjuvant Breast Project B-04 study demonstrated survival equivalence for operable, clinically node-negative breast cancer patients managed by axillary surgery versus axillary irradiation versus observation and delayed therapeutic ALND at the time of regional failure. This outcome equivalence has persisted on 25-year follow-up. ${ }^{1}$ Despite these findings, the ALND remained an essential component of breast cancer management because the axillary nodal status became the most powerful determinant of the magnitude of benefit from adjuvant systemic therapy. Furthermore, the B-04 study was not powered statistically to address the survival benefits of the ALND; it was designed to evaluate the overall safety of modified surgical strategies (radical mastectomy vs. total mastectomy vs. total mastectomy and locoregional irradiation) as treatment for operable breast cancer. Accrual to this phase III clinical trial was completed in an era of surgical treatment alone for breast cancer management, before the advent of effective systemic therapy for breast cancer.

A standard level I/II ALND has therefore been routinely performed in patients with invasive breast 
TABLE 4. Multivariate model predictions for the probability of a positive case

\begin{tabular}{lcccc}
\hline Positive SLNs $^{a}$ & Tumor size $(\mathrm{cm})$ & Lymphovascularinvasion & Extranodal extension & Predicted probability \\
\hline 1 & $\leq 2$ & Absent & Absent & .002 \\
2 & $\leq 2$ & Absent & Absent & .070 \\
3 & $\leq 2$ & Absent & Absent & .267 \\
1 & $>2$ & Absent & Absent & .020 \\
2 & $>2$ & Absent & Absent & .197 \\
3 & $>2$ & Absent & Absent & .541 \\
1 & $\leq 2$ & Present & Absent & .017 \\
2 & $\leq 2$ & Present & Absent & .177 \\
3 & $\leq 2$ & Present & Absent & .510 \\
1 & $\leq 2$ & Absent & Present & .018 \\
2 & $\leq 2$ & Absent & Present & .182 \\
3 & $\leq 2$ & Absent & Absent & .518 \\
1 & $>2$ & Present & Absent & .055 \\
2 & $>2$ & Present & Absent & .411 \\
3 & $>2$ & Present & Present & .771 \\
1 & $>2$ & Absent & Present & .057 \\
2 & $>2$ & Absent & Present & .419 \\
3 & $>2$ & Absent & Present & .776 \\
1 & $\leq 2$ & Present & Present \\
2 & $\leq 2$ & Present & Present & .050 \\
3 & $\leq 2$ & Present & Present & .389 \\
1 & $>2$ & Present & Present & .754 \\
3 & $>2$ & Present & Present & .146 \\
.673 \\
.908 \\
\hline
\end{tabular}

SLN, sentinel lymph node.

${ }^{a}$ The total number of SLNs removed was fixed at 3 .

cancer, but several issues have motivated efforts to modify this strategy. First, ALND patients face a lifelong risk of lymphedema that ranges from $10 \%$ to $50 \%,{ }^{12}$ depending on other risk factors, the duration of follow-up, and the method of detection. Other morbidities associated with an ALND include neurosensory disturbances and shoulder dysfunction. ${ }^{13}$ Also, the successful implementation of breast cancer screening programs has resulted in detection of high proportions of microinvasive and $\mathrm{Tla}$ tumors that are more likely to be node negative, but these patients have nonetheless faced the morbidity of the ALND to have definitive proof of their nodenegative status.

The introduction of intraoperative lymphatic mapping and SLN biopsy programs for breast cancer patients ${ }^{14,15}$ was followed by the rapid adoption of this technology to stage newly diagnosed cases. ${ }^{16}$ Many programs now routinely accept a negative SLN as accurate, and these patients are spared the morbidity of the completion ALND. Several studies with long-term follow-up ${ }^{17-20}$ are now reporting excellent results with this strategy, as well as improved quality of life. The SLN biopsy therefore screens newly diagnosed breast cancer patients for nodal metasta- ses, and only SLN-positive patients proceed to undergo completion ALND.

Ongoing controversies regarding the survival benefits of the standard ALND in patients with a clinically benign axilla are therefore revisited in the era of SLN biopsy. Once the presence versus absence of nodal metastases is confirmed histopathologically and this information is appropriately incorporated into decision algorithms for adjuvant systemic therapy, it can be argued that the completion ALND is unnecessary. As suggested by the National Surgical Adjuvant Breast Project B-04 study, clinically occult and untreated axillary metastases will progress into clinically evident disease that necessitates a delayed therapeutic ALND in approximately half of cases, and this outcome does not seem to adversely affect survival when compared with patients whose axillary disease was detected by a staging ALND at the time of diagnosis. ${ }^{1}$ Overall, $19 \%$ of the patients randomized to total mastectomy alone experienced an axillary relapse as an initial treatment failure.

The risk of regional failure is likely to be substantially lower in contemporary practice, where the node-positive patient is identified by an SLN biopsy. The standard of care for these patients with stage II 
disease will include adjuvant systemic therapy, which will contribute to control of regional disease. ${ }^{21}$ Also, studies of patients undergoing SLN biopsy with a concomitant ALND have demonstrated that axillary metastases will be limited to the SLN in $30 \%$ to $67 \%$ of cases. ${ }^{8,15,22-24}$ In these cases, the completion ALND subjects the patient to additional surgery and morbidity to remove negative non-SLNs.

The American College of Surgeons Oncology Group Z0011 protocol was a prospective, randomized trial specifically designed to answer the question of whether the completion ALND will improve outcome in SLN-positive patients, by randomizing these cases to standard axillary surgery versus axillary observation. The unfortunate recent closure of this trial because of slow accrual rates (but not because of adverse event rates, which have thus far been low in both the ALND and axillary observation arms) leaves this question unanswered and has strengthened the need for alternative strategies that can improve our ability to determine which SLN-positive patients are likely to benefit from the completion ALND.

Van Zee et al. $^{4}$ therefore developed a nomogram that estimates the likelihood that an individual SLNpositive patient will have additional metastatic nodes in the completion ALND specimen. Degnim et al. ${ }^{5}$ conducted a meta-analysis of studies involving SLN biopsy with concomitant ALND, and this pooled analysis provided a robust assessment of the clinicopathologic features associated with the likelihood of detecting metastatic disease in non-SLNs. Both of these investigators have found primary tumor size and extent of SLN pathology to be strong predictors of non-SLN disease.

Accurate identification of patients with any metastatic non-SLNs is a potentially valuable strategy, but it is most important for the surgeon to identify the subset of high-risk cases in which adjuvant therapy (and possibly survival) will be influenced by the pathology findings from the completion ALND specimen. Patients with one, two, or three metastatic lymph nodes will receive "standard" adjuvant systemic therapy for node-positive disease, and adjuvant radiation will be delivered on the basis of the primary operation (lumpectomy vs. mastectomy) and primary tumor size. In contrast, patients with high-risk disease defined by the presence of four or more metastatic lymph nodes will be referred for extended-field irradiation to the chest wall and nodal basins. ${ }^{6,7}$ As shown in Table 5, approximately $10 \%$ to $16 \%$ of SLN-positive breast cancer patients will fall into this high-risk category, and these patients clearly benefit from aggressive surgical and radiotherapeutic ap-
TABLE 5. Proportion of SLN-positive breast cancer patients found to have at least four metastatic nodes after SLN biopsy and completion ALND (metastatic SLNS and non-SLNS)

\begin{tabular}{lcc}
\hline Study & No. of cases & $\begin{array}{c}\text { \% Cases with } \\
\text { nodal metastases }\end{array}$ \\
\hline Krag $^{8}$ & 114 & 16 \\
Wong $^{9}$ & 389 & 13 \\
Chu $^{1}$ & 158 & 11 \\
Abdessalam $^{11}$ & 100 & 12 \\
Shahar $^{25}$ & 265 & 11 \\
This study & 285 & 14 \\
\hline
\end{tabular}

SLN, sentinel lymph node; ALND, axillary lymph node dissection.

proaches aimed at optimizing locoregional control of disease. We therefore sought to develop a model based on the clinicopathologic features studied by Van Zee et al. ${ }^{4}$ and Degnim et al. ${ }^{5}$ to specifically identify SLN-positive patients with extensive axillary nodal disease.

Shahar et al. ${ }^{25}$ addressed this issue as well in their study from the University of Texas M. D. Anderson Cancer Center, where the following features were predictive of having four or more metastatic nodes after a positive SLN biopsy: no drainage seen on lymphoscintigraphy, more than one positive SLN, and lymphovascular space invasion. The M. D. Anderson study was based on 28 high-risk patients $(10.5 \%)$ identified from 265 SLN-positive patients. We sought to expand on this work by studying a more robust dataset composed of patients treated at the M. D. Anderson Cancer Center and the University of Michigan Comprehensive Cancer Center. This approach yielded 41 cases with at least 4 metastatic nodes (14\% of the total 285-patient population).

Our bivariate comparisons revealed that the size of the metastatic focus in the SLN was strongly correlated with the risk of having high-risk axillary disease. Of the 74 patients with micrometastatic disease in the SLN (focus up to $2 \mathrm{~mm}$ in diameter or detected by immunohistochemistry), only 1 (1.4\%) had extensive nodal disease, versus 36/194 (19\%) patients with metastatic foci $>2 \mathrm{~mm}(P<.0001)$. This feature was one of the most powerful predictors identified in the pooled analysis by Degnim et al. ${ }^{5}$ as well. Logistic regression, however, revealed that the size of the metastatic focus was no longer an independently predictive variable. The model that we developed was therefore based on the primary tumor size, the volume of SLN metastases, and the presence versus absence of lymphovascular invasion and extranodal extension. These characteristics retained significance in our multivariate analysis. 
Our multivariate model also indicated that the percentage of metastatic SLNs (the number of metastatic SLNs divided by the total number of SLNs resected multiplied by 100) was significant as a continuous covariate (odds ratio, 1.03 ; 95\% confidence interval, 1.01-1.05; $P=.0001)$. For the presentation of predicted probabilities listed in Table 4, we chose to fix the total number of SLNs removed at the median value of 3 to focus the reader's attention on the contribution of the absolute number of metastatic SLNs. The proportion of metastatic SLNs to the total number of SLNs resected will vary substantially with differences in the sentinel nodal drainage pattern and surgical specimen. If the total number of SLNs removed was $>3$, the percentage of metastatic to total SLNs would be reduced in our prediction model; hence, the predicted probabilities listed in Table 4 would be also be reduced. For an example, if we consider two identical patients, each with a T1/T2 tumor and without lymphovascular invasion or extranodal extension, then a patient with only a single SLN removed that is also metastatic will have a percentage of $100 \%$, whereas a patient with only 1 metastatic node out of a total of 10 SLNs resected will have a percentage of only $10 \%$. The predicted probability of having high-risk axillary disease is $4.6 \%$ for the former example and only .3\% for the latter. This difference illustrates that by including the percentage of metastatic SLNs in our final multivariate model, we are correctly accounting for the extent of axillary sampling that has been achieved by the sentinel lymphadenectomy. Intuitively, a larger extent of the sentinel drainage area, the involved nodes, and the resultant sentinel lymphadenectomy, without the discovery of four or more metastatic nodes, reduces the number of nodes remaining in the axilla that could be harboring metastatic disease, thus reducing a patient's probability of having extensive axillary disease.

In summary, our study of SLN-positive breast cancer yielded a straightforward and biologically plausible strategy for assessing the risk that a patient will be found to have a total metastatic burden of four or more involved lymph nodes. Our model revealed a $.2 \%$ likelihood that a completion ALND will convert a case with a T1 tumor and a single positive SLN that lacks both lymphovascular invasion and extranodal extension into a case of extensive nodal disease. The likelihood of having highrisk pathologic characteristics identified on completion ALND can be estimated by evaluating the primary tumor size, volume of metastatic SLNs, and presence versus absence of adverse histopathologic features. A completion ALND remains the standard of care for patients who have a metastatic SLN, but the ability to predict the likelihood that this ALND will affect subsequent management can be helpful in cases in which the patient or the multidisciplinary oncology team is reluctant pursue additional surgery. Our results require validation by other investigators.

\section{ACKNOWLEDGMENT}

The authors gratefully acknowledge support for this study from the Ruth Glancy Breast Cancer Research Fund.

\section{REFERENCES}

1. Fisher B, Jeong JH, Anderson S, et al. Twenty-five-year followup of a randomized trial comparing radical mastectomy, total mastectomy, and total mastectomy followed by irradiation. $N$ Engl J Med 2002; 347:567-75.

2. Wilke LG, Giuliano A. Sentinel lymph node biopsy in patients with early-stage breast cancer: status of the National Clinical Trials. Surg Clin North Am 2003; 83:901-10.

3. Wells S, Giuliano A, Hunt K. ACOSOG Z0011 suspension of registration. American College of Surgeons Oncology Group, 2004. Available: www.acosog.org [accessed December 2, 2005].

4. van Zee KJ, Manasseh DM, Bevilacqua JL, et al. A nomogram for predicting the likelihood of additional nodal metastases in breast cancer patients with a positive sentinel node biopsy. Ann Surg Oncol 2003; 10:1140-51.

5. Degnim AC, Griffith KA, Sabel MS, et al. Clinicopathologic features of metastasis in nonsentinel lymph nodes of breast carcinoma patients. Cancer 2003; 98:2307-15.

6. Recht A, Edge SB, Solin LJ, et al. Postmastectomy radiotherapy: clinical practice guidelines of the American Society of Clinical Oncology. J Clin Oncol 2001; 19:1539-69.

7. Recht A, Edge SB. Evidence-based indications for postmastectomy irradiation. Surg Clin North Am 2003; 83:995-1013.

8. Krag D, Weaver D, Ashikaga T, et al. The sentinel node in breast cancer - a multicenter validation study. $N$ Engl J Med 1998; 339:941-6.

9. Wong SL, Edwards MJ, Chao C, et al. Predicting the status of the nonsentinel axillary nodes: a multicenter study. Arch Surg 2001; 136:563-8.

10. Chu KU, Turner RR, Hansen NM, et al. Do all patients with sentinel node metastasis from breast carcinoma need complete axillary node dissection? Ann Surg 1999; 229:536-41.

11. Abdessalam SF, Zervos EE, Prasad M, et al. Predictors of positive axillary lymph nodes after sentinel lymph node biopsy in breast cancer. Am J Surg 2001; 182:316-20.

12. Erickson VS, Pearson ML, Ganz PA, et al. Arm edema in breast cancer patients. J Natl Cancer Inst 2001; 93:96-111.

13. Engel J, Kerr J, Schlesinger-Raab A, et al. Axilla surgery severely affects quality of life: results of a 5-year prospective study in breast cancer patients. Breast Cancer Res Treat 2003; 79:47-57.

14. Krag DN, Weaver DL, Alex JC, et al. Surgical resection and radiolocalization of the sentinel lymph node in breast cancer using a gamma probe. Surg Oncol 1993; 2:335-9; discussion 340 .

15. Giuliano AE, Kirgan DM, Guenther JM, et al. Lymphatic mapping and sentinel lymphadenectomy for breast cancer. Ann Surg 1994; 220:391-8; discussion 398-401. 
16. Zavagno G, Salvo GL, Casara D, et al. Sentinel node biopsy for breast cancer: is it already a standard of care? A survey of current practice in an Italian region. BMC Cancer 2004; 4:2.

17. Torrenga H, Fabry H, Van der Sijp JR, et al. Omitting axillary lymph node dissection in sentinel node negative breast cancer patients is safe: a long term follow-up analysis. J Surg Oncol 2004; 88:4-7discussion 7-8.

18. Rietman JS, Dijkstra PU, Geertzen JH, et al. Treatment-related upper limb morbidity 1 year after sentinel lymph node biopsy or axillary lymph node dissection for stage I or II breast cancer. Ann Surg Oncol 2004; 11:1018-24.

19. Reitsamer R, Peintinger F, Prokop E, et al. 200 Sentinel lymph node biopsies without axillary lymph node dissection-no axillary recurrences after a 3-year follow-up. Br J Cancer 2004; 90:1551-4.

20. Veronesi U, Paganelli G, Viale G, et al. A randomized comparison of sentinel-node biopsy with routine axillary dissection in breast cancer. $N$ Engl $J$ Med 2003; 349:546-53.
21. Kuerer HM, Sahin AA, Hunt KK, et al. Incidence and impact of documented eradication of breast cancer axillary lymph node metastases before surgery in patients treated with neoadjuvant chemotherapy. Ann Surg 1999; 230:72-8.

22. Giuliano AE, Jones RC, Brennan M, et al. Sentinel lymphadenectomy in breast cancer. J Clin Oncol 1997; 15:2345-50.

23. Borgstein PJ, Pijpers R, Comans EF, et al. Sentinel lymph node biopsy in breast cancer: guidelines and pitfalls of lymphoscintigraphy and gamma probe detection. $J$ Am Coll Surg 1998; 186:275-83.

24. Albertini JJ, Lyman GH, Cox C, et al. Lymphatic mapping and sentinel node biopsy in the patient with breast cancer. JAMA 1996; 276:1818-22.

25. Shahar KH, Hunt KK, Thames HD, et al. Factors predictive of having four or more positive axillary lymph nodes in patients with positive sentinel lymph nodes: implications for selection of radiation fields. Int J Radiat Oncol Biol Phys 2004; 59:1074-9. 